(0) The Journal of Risk and Insurance, 2005, Vol. 72, No. 4, 577-599

\title{
On the Consequences of Behavioral ADAPTATIONS IN tHe COST-BeNefit ANALYSIS Of ROAd SAFETY MEASURES
}

\author{
Olivier Gossner \\ Pierre Picard
}

\begin{abstract}
It is sometimes argued that road safety measures or automobile safety standards fail to save lives because safer highways or safer cars induce more dangerous driving. A similar but less extreme view is that ignoring the behavioral adaptation of drivers would bias the cost-benefit analysis of a traffic safety measure. This article derives cost-benefit rules for automobile safety regulation when drivers may adapt their risk-taking behavior in response to changes in the quality of the road network. The focus is on the financial externalities induced by accidents because of the insurance system as well as on the consequences of drivers' risk aversion. We establish that road safety measures are Pareto improving if their monetary cost is lower than the difference between their (adjusted for risk aversion) direct welfare gain with unchanged behavior and the induced variation in insured losses due to drivers' behavioral adaptation. The article also shows how this rule can be extended to take other accident external costs into account.
\end{abstract}

\section{INTRODUCTION}

Behavioral adaptations of drivers to safety measures is a well-documented phenomenon; see OECD (1990). Following changes in the road-vehicle system affecting either highways safety or vehicle safety, road users sometimes adapt their behavior in a manner inconsistent with the initial goals of the safety measures: indeed, safer highways and safer cars often induce more dangerous driving. ${ }^{1}$ Because of this behavioral adaptation, the decrease in accidents and fatalities may be lower than what

Olivier Gossner is at Paris-Jourdan Sciences Economiques. Pierre Picard is at Ecole Polytechnique and HEC (Paris). The author can be contacted via e-mail: pierre.picard@polytechnique. edu. The authors thank Georges Dionne, James Hammitt, Henri Loubergé, and the two referees for helpful comments. Financial support from FFSA is gratefully acknowledged.

${ }^{1}$ See, for instance, the analysis of Peterson, Hoffer, and Millner (1995) on the behavior of drivers of air-bag-equipped cars. They conclude that "air-bag-equipped cars tend to be driven more aggressively than cars without air-bags and that the additional aggressiveness appears to offset the effect of the air-bag for the driver and increases the risk of death to others." 
was initially expected, and it may even fully vanish. In particular, using US data (1947-1972), Peltzman (1975) claimed that such offsetting effects have been virtually complete and that regulation has not decreased highway deaths. However, most studies lead to less clear-cut conclusions and they most frequently show that behavioral adaptation only mitigates the initial impact of road safety measures and that these measures still entail some residual effects. ${ }^{2}$

Behavioral adaptation to safety measures has been widely analyzed with the analytical tools of social psychology and cognition theory. In particular, Wilde (1982, 2001) develops a theory of risk homeostasis according to which individuals are prepared to accept a given target level of risk and he derives a number of implications for safety and health. In Wilde's theory, the dynamics of individual behavior results from a gap between the perceived level of risk and an (exogenously given) target level of risk. This gap follows from the fact that individuals have bounded perceptual skills. However, if the risk-taking behavior by drivers is put within the framework of expected utility maximization (for instance by speed choice), then risk homeostasis is possible only under very restrictive conditions; see Janssen and Tenking (1988). Nevertheless, behavioral adaptations to changes in the road transport system is a much more general phenomenon than risk homeostasis which is expected to hold under fairly general conditions. The fact that compensation is partial or total (or even that there is overcompensation) is a matter that can be settled on the basis of empirical observations only. As summarized in the key conclusions of an OECD (1990) expert group report "Behavioral adaptation (to changes in the road transport system) exists, and does have an effect on the safety benefits achieved through road safety programs. Results indicate that, generally, behavioral adaptation does not eliminate the safety gains obtained, but it does reduce the effectiveness of road safety programs in a number of cases."

Given the empirical fact that drivers adapt their behavior to changes in their environment (particularly to road safety measures), an open question is how these behavioral adaptations should be taken into account in the cost-benefit analysis of public investments and traffic regulation. In particular, behavioral adaptation may totally or partially offset the safety gains of an investment transport program. These adaptations may correspond to a welfare gain to individuals, for instance, when individuals enjoy a more pleasant driving or chose a higher mileage, even if these side effects were not the main goal of the program. So, one may actually wonder whether and how to include these adaptations in the balance of costs and benefits of road safety programs.

The following analysis will bring insurance-linked externalities into prominence. Indeed, we must keep in mind that drivers are (at least partially) insured against the losses they may cause when they are responsible for an accident. Reckless drivers not only take a risk for themselves but, when they bring about an accident, they are also the source of an externality to the detriment of all other drivers because of the

\footnotetext{
${ }^{2}$ Graham and Garber (1984) critique the functional form of Peltzman's model and, saying the opposite of what Peltzman says, they argue that "safety standards have saved tens of thousands of lives during the 1970s." Later on, works by Chirunko and Harper (1993) and Keeler (1994) have reinforced the empirical plausibility of the "offsetting behavior hypothesis."
} 
insurance system. One may even say that the essence of insurance contracting under moral hazard is to trade off the incentives for drivers to be more cautious in order to reduce this detrimental external effect with the positive effect of a better risk sharing between drivers. From our analysis, it emerges that the insurance mechanism is the fundamental reason why behavioral adaptations should be taken into account by the social planner: the social cost of these adaptations is nothing but the additional burden imposed on the drivers as a whole by this insurance-linked externality.

Blomquist (1988, pp. 32-39) emphasizes the fact that individual demand for traffic safety tends to be too low because of such insurance external effects, which include "the potential shift of some medical costs to those not involved in an accident, ..., the lack of precise experience rating for automobile insurance and possibly incomplete compensation for damaged parties through the courts especially when time costs and death are involved." He also draws attention to the fact that conventional cost-benefit analysis assumes that drivers are passive toward road safety measure (for instance, mandatory safety standards) and that they do not change their safety-related behavior. He concludes that the assumption of driver's passivity biases the conventional costbenefit studies toward positive net benefits of an active traffic safety policy. In what follows, we will show that both issues (insurance external effects and behavioral adaptation) are in fact tightly connected: forgetting the behavioral adaptations biases the cost-benefit analysis in so far as the risk-taking behaviors by some drivers make the other drivers incur additional insurance costs.

In order to develop such an analysis, we will insert the risk-taking behavior by drivers within a simple one-good model of optimal resource allocation under uncertainty with insurance coverage. This setting will allow us to identify the way in which the conventional cost-benefit rules should be rectified because of drivers' behavioral adaptation. It will also highlight the effect of drivers' risk aversion on the willingness to pay for a traffic safety measure.

Finally, car crashes may entail additional costs incurred by other individuals than those who are directly involved in the accidents, leading to a difference between the private costs and the social costs of accidents. This difference may be substantial and we will see how it interferes with the cost-benefit analysis of road safety measures.

From now on, two shortcomings of the present article should be acknowledged. First, for the sake of clarity, we will only focus attention on the effect of road safety measures on drivers' risk-taking behavior. However, a road safety policy may also induce an increase in the density of traffic and, in consequence, to additional congestion costs or to an increase in average accident risk. ${ }^{3}$ In practice, a thorough cost-benefit analysis should not overlook these externalities which take place through the effect of road safety measures on traffic density. Secondly, we will only consider network-related public expenditures, be they preventive (e.g., lane widening, line marking, lighting of freeways, ... ) or protective (e.g., crash barriers). Vehicle safety will not be approached although it is an essential part of many road safety policies. Our results can easily be extended to the analysis of vehicle-related mandatory restraints (e.g., more restrictive

\footnotetext{
${ }^{3}$ On the fact that the density of traffic affects average accident risk; see, for instance, Jansson (1994) and Small and Gomez-Ibanez (1999).
} 
crash tests or more efficient antilocking systems) when drivers may adapt their risktaking behavior in reaction to changes in regulation. ${ }^{4}$

The article is organized as follows: The second section presents the basic model of drivers' behavior with accident risk. The third section develops the costs-benefits analysis of road safety programs when drivers change their safety-related behavior in reaction to changes in public investments. The last section concludes.

\section{A Microeconomic Model of Accident Risk}

In this section, we analyze a simple model of road users' behavior. Assume that there are $n$ drivers, indexed by $i=1, \ldots, n$. A typical driver is treated as an expected utility maximizer. The utility of driver $i$ depends on final random wealth $w_{i}^{f}$ and effort $e_{i}$. It is written as

$$
u_{i}\left(w_{i}^{f}, e_{i}\right)
$$

with $\partial u_{i} / \partial w_{i}^{f}>0, \partial^{2} u_{i} / \partial w_{i}^{f 2}<0, \partial u_{i} / \partial e_{i}<0, \partial^{2} u_{i} / \partial e_{i}^{2} \leq 0$. Hence drivers are risk averse with respect to wealth, they dislike effort and their marginal disutility of effort is nondecreasing. ${ }^{5}$

This may be interpreted in two different ways. Effort may be nonmonetary (driving cautiously) or monetary (buying a safer car). The principal-agent literature frequently assumes that effort is nonmonetary and in that case, it is often postulated that utility is separable in wealth and effort. If effort is directly measured by its disutility, we then have

$$
u_{i}\left(w_{i}^{f}, e_{i}\right) \equiv v_{i}\left(w_{i}^{f}\right)-e_{i}
$$

with $v_{i}^{\prime}>0, v_{i}^{\prime \prime}<0$. Such a separability assumption makes the analysis easier, but we may find it oversimplified. An increase in wealth may well make effort more painful to the driver and in that case, we have $\partial^{2} u_{i} / \partial w_{i}^{f} \partial e_{i}<0$. This will be the case, for example, when making more effort means abiding by the speed limits and the private value of time is positively correlated with wealth.

When effort is monetary, we may identify $e_{i}$ with expenditures which gives

$$
u_{i}\left(w_{i}^{f}, e_{i}\right) \equiv v_{i}\left(w_{i}^{f}-e_{i}\right)
$$

with once again $v_{i}^{\prime}>0, v_{i}^{\prime \prime}<0$. We then have $\partial^{2} u_{i} / \partial w_{i}^{f} \partial e_{i}=-v_{i}^{\prime \prime}>0$.

In the general case, the cross-derivative $\partial^{2} u_{i} / \partial w_{i}^{f} \partial e_{i}$ may thus be nil, positive, or negative.

\footnotetext{
${ }^{4}$ Such an extension can be obtained from the authors on request.

${ }^{5}$ Utility is supposed to be state independent. In a state dependent approach-Dionne (1982) and Arnott (1992) — the utility function would depend on whether an accident occurs or not.
} 
Let $p_{i}$ denote the probability of an accident caused by driver $i$. It depends on drivers $i$ 's effort $e_{i}$ and also on a parameter $x$ that describes the safety of the road network. Hence, we will write

$$
p_{i}=p_{i}\left(e_{i}, x\right)
$$

with $\partial p / \partial e_{i}<0, \partial^{2} p / \partial e_{i}^{2}>0$, and $\partial p / \partial x<0$. Changes in $x$ reflect the modifications in the highways insofar as they affect the probability of accident for a given behavior of the driver. For notational simplicity, we assume that all drivers are affected by the same road transport system parameter $x$ and that the relation between the probability of an accident and the effort level is the same for all drivers. A preventive road safety measure corresponds to an increase in $x$.

Let $\tilde{y}_{i}$ be the damages (in monetary terms) caused by driver $i$ in the event of an accident: $\tilde{y}_{i}$ is a random variable that corresponds to the total cost of an accident, including damages inflicted on the other road users. For the time being, we assume that accidents only entail private costs borne by the individuals involved in the crashes. We will later extend our results to the more general case where accidents entail external costs. For simplicity, we assume that the probability distribution of $\tilde{y}_{i}$ does not depend on $e_{i}$. In other words, the probability of an accident depends on the driver's effort but, should an accident occur, then the amount of losses is independent of it. Our analysis extends straightforwardly to the (more realistic case) where the risk-taking behavior also affects the probability distribution of damages, conditionally on an accident occurring. The probability distribution of $\tilde{y}_{i}$ is the same for all $i$ and we restrict attention to a set of random variables $\tilde{y}_{i}$ that can be completely ordered by first-order stochastic dominance. We index these probability distributions by their mean value $y=\mathbf{E} \tilde{y}_{i}$ for all $i .^{6}$ Hence, an increase in $y$ corresponds to a first-order dominance shift in $\tilde{y}_{i}$. A protective road safety measure corresponds to a decrease in $y$.

Let $\tilde{\ell}_{i}$ be the random cost of an accident to the at fault driver $i$ and his or her family. $\tilde{\ell}_{i}$ depends on $\tilde{y}_{i}$ and also on a random noise $\varepsilon_{i}$ which reflects the specificity of the accident. We write

$$
\tilde{\ell}_{i}=\ell_{i}\left(\tilde{y}_{i}, \varepsilon_{i}\right)
$$

with $\partial \ell_{i} / \partial \tilde{y}_{i} \geq 0$, where $\varepsilon_{i}$ is a random variable which reflects the specificity of the accidents. Function $\ell_{i}\left(\tilde{y}_{i}, \varepsilon_{i}\right)$ implicitly depends on the insurance coverage. For instance, in case of a deductible, we have $\partial \ell_{i} / \partial \tilde{y}_{i}=1$ for small $\tilde{y}_{i}$. Conversely, $\partial \ell_{i} / \partial \tilde{y}_{i}=0$ if there is full insurance at the margin, and $0<\partial \ell_{i} / \partial \tilde{y}_{i}<1$ in case of coinsurance. Furthermore, the loss incurred by the driver also depends on the type of the accident. For instance, full liability insurance is usually required but drivers may have partial insurance for the damages to their own car. Likewise, there may be partial bodily injury insurance (or even no pretium doloris insurance) and full property insurance.

\footnotetext{
${ }^{6}$ More realistically, E $\tilde{y}_{i}$ may depend on parameters which are differentiated among drivers, such as the size of the family or the type of the car. The model could be easily extended in this direction by defining $y$ as a parameter that affects the expected accident damages, i.e., $\mathbf{E} \tilde{y}_{i}=\Phi_{i}(y)$ with $\Phi_{i}^{\prime}>0$.
} 
The $\ell_{i}(.$, .) function are taken exogenously given. They may differ among drivers, particularly when drivers buy different insurance policies with more or less generous coverage. Given $\ell_{i}(.,$.$) and \varepsilon_{i}$, the probability distribution of $\tilde{\ell}_{i}$ depends on $y$ and because $\partial \ell_{i} / \partial \tilde{y}_{i} \geq 0$, an increase in $y$ entails a first-order dominance shift in $\tilde{\ell}_{i}$.

Let $w_{i}$ be the wealth of driver $i$ in the no-accident state. We have

$$
w_{i}=\bar{w}_{i}-P_{i}-t_{i}
$$

where $\bar{w}_{i}$ denotes driver $i$ 's initial wealth, $P_{i}$ denotes the insurance premium, and $t_{i}$ is a (positive or negative) transfer paid by driver $i$ to the government. $\bar{w}_{i}$ is taken as exogenously given. Determinants of $P_{i}$ and $t_{i}$ will be clarified in the next section.

We assume full liability of drivers for the damages they may inflict on other drivers. Hence, the final wealth of a driver is unaffected if he or she is the victim of an accident caused by another driver. From a descriptive standpoint, this is certainly an oversimplification of reality as it amounts to assuming that all damages can be compensated. We make this assumption since partial liability would make the modeling more cumbersome without any qualitative change in the results. Hence we have $w_{i}^{f}=$ $w_{i}$ if driver $i$ does not cause any accident and $w_{i}^{f}=w_{i}-\tilde{\ell}_{i}$ in the event of an accident caused by this driver.

Driver $i$ maximizes

$$
U_{i}\left(e_{i}, x, y, w_{i}\right) \equiv\left[1-p\left(e_{i}, x\right)\right] u_{i}\left(w_{i}, e_{i}\right)+p\left(e_{i}, x\right) \mathbf{E}\left[u_{i}\left(w_{i}-\tilde{\ell}_{i}, e_{i}\right) \mid y\right]
$$

with respect to $e_{i} \geq 0$. Let $\hat{e}_{i}=\hat{e}_{i}\left(x, y, w_{i}\right)$ be the optimal level of effort chosen by the driver and $p_{i}=p\left(\hat{e}_{i}, x\right)$. The first-order condition for the optimal choice of effort is

$$
\begin{aligned}
\frac{\partial U_{i}\left(\hat{e}_{i}, x, y, w_{i}\right)}{\partial e_{i}}= & -\frac{\partial p\left(\hat{e}_{i}, x\right)}{\partial e_{i}}\left[u_{i}\left(w_{i}, \hat{e}_{i}\right)-\mathbf{E}\left[u_{i}\left(w_{i}-\tilde{\ell}_{i}, \hat{e}_{i}\right) \mid y\right]\right] \\
& +\left(1-p_{i}\right) \frac{\partial u_{i}\left(w_{i}, \hat{e}_{i}\right)}{\partial e_{i}}+p_{i} \frac{\partial \mathbf{E}\left[u_{i}\left(w_{i}-\tilde{\ell}_{i}, \hat{e}_{i}\right) \mid y\right]}{\partial e_{i}}=0
\end{aligned}
$$

if $\hat{e}_{i}>0$, which will be assumed in what follows. We have

$$
\begin{aligned}
\frac{\partial^{2} U_{i}\left(e_{i}, x, y, w_{i}\right)}{\partial e_{i}^{2}}= & -\frac{\partial^{2} p\left(e_{i}, x\right)}{\partial e_{i}^{2}}\left[u_{i}\left(w_{i}, e_{i}\right)-\mathbf{E}\left[u_{i}\left(w_{i}-\tilde{\ell}_{i}, e_{i}\right) \mid y\right]\right] \\
& -2 \frac{\partial p\left(e_{i}, x\right)}{\partial e_{i}}\left[\frac{\partial u_{i}\left(w_{i}, e_{i}\right)}{\partial e_{i}}-\frac{\partial \mathbf{E}\left[u_{i}\left(w_{i}-\tilde{\ell}_{i}, e_{i}\right) \mid y\right]}{\partial e_{i}}\right] \\
& +\left[1-p\left(e_{i}, x\right)\right] \frac{\partial^{2} u_{i}\left(w_{i}, e_{i}\right)}{\partial e_{i}^{2}}+p_{i} \frac{\partial^{2} \mathbf{E}\left[u_{i}\left(w_{i}-\tilde{\ell}_{i}, e_{i}\right) \mid y\right]}{\partial e_{i}^{2}}
\end{aligned}
$$

The terms in the first and third lines of Equation (4) are negative. The term in the second line is nonpositive when $\partial^{2} u_{i} / \partial w_{i}^{f} \partial e_{i} \leq 0$, which includes the case of a separable utility function. In such a case, $U_{i}$ is strictly concave with respect to $e_{i}$. However, when 
$\partial^{2} u_{i} / \partial w_{i}^{f} \partial e_{i}>0$, then the term in the second line of (4) is positive and $U_{i}$ may not be a concave function of $e_{i}$. Consequently, $\hat{e}_{i}\left(x, y, w_{i}\right)$ may have some discontinuity points.

At any continuity point, the local second-order condition is

$$
A \equiv \frac{\partial^{2} U_{i}\left(\hat{e}_{i}, x, y, w_{i}\right)}{\partial e_{i}^{2}}<0
$$

Differentiating (3) then gives

$$
\begin{aligned}
\frac{\partial \hat{e}_{i}}{\partial x}=\frac{1}{A}\{ & \frac{\partial^{2} p\left(\hat{e}_{i}, x\right)}{\partial e_{i} \partial x}\left[u_{i}\left(w_{i}, \hat{e}_{i}\right)-\mathbf{E}\left[u_{i}\left(w_{i}-\tilde{\ell}_{i}, \hat{e}_{i}\right) \mid y\right]\right] \\
& \left.\times \frac{\partial p\left(\hat{e}_{i}, x\right)}{\partial x}\left[\frac{\partial u_{i}\left(w_{i}, \hat{e}_{i}\right)}{\partial e_{i}}-\frac{\partial \mathbf{E}\left[u_{i}\left(w_{i}-\tilde{\ell}_{i}, \hat{e}_{i}\right) \mid y\right]}{\partial e_{i}}\right]\right\}, \\
\frac{\partial \hat{e}_{i}}{\partial y}=-\frac{1}{A}\left\{\frac{\partial p\left(\hat{e}_{i}, x\right)}{\partial e_{i}} \frac{\partial \mathbf{E}\left[u_{i}\left(w_{i}-\tilde{\ell}_{i}, \hat{e}_{i}\right) \mid y\right]}{\partial y}+p_{i} \frac{\partial^{2} \mathbf{E}\left[u_{i}\left(w_{i}-\tilde{\ell}_{i}, \hat{e}_{i}\right) \mid y\right]}{\partial e_{i} \partial y}\right\}, & \\
\frac{\partial \hat{e}_{i}}{\partial w_{i}}=\frac{1}{A}\left\{\frac{\partial p\left(\hat{e}_{i}, x\right)}{\partial e_{i}}\left[\frac{\partial u_{i}\left(w_{i}, \hat{e}_{i}\right)}{\partial w_{i}^{f}}-\frac{\partial \mathbf{E}\left[u_{i}\left(w_{i}-\tilde{\ell}_{i}, \hat{e}_{i}\right) \mid y\right]}{\partial w_{i}^{f}}\right]\right. & \left.-\left(1-p_{i}\right) \frac{\partial^{2} u_{i}\left(w_{i}, \hat{e}_{i}\right)}{\partial e_{i} \partial w_{i}^{f}}-p_{i} \frac{\partial^{2} \mathbf{E}\left[u_{i}\left(w_{i}-\tilde{\ell}_{i}, \hat{e}_{i}\right) \mid y\right]}{\partial e_{i} \partial w_{i}^{f}}\right\} .
\end{aligned}
$$

These equations lead us to clear-cut conclusions when utility is separable between wealth and effort. Indeed, when $\partial^{2} u_{i} / \partial w_{i}^{f} \partial e_{i}=0$, then several terms vanish in (5), (6) and (7) and we can sign the partial derivatives of $\hat{e}_{i}\left(x, y, w_{i}\right)$. First, we may observe that $\partial \hat{e}_{i} / \partial x$ and $\partial^{2} p / \partial e_{i} \partial x$ have opposite signs. In particular, when an increase in $x$ reduces the marginal effect of the driver's effort on the probability of accident, i.e., when $\partial^{2} p / \partial e_{i} \partial x>0$, then $\partial \hat{e}_{i} / \partial x$ is negative. In such a case, the safer the road network, the lower the marginal benefit of effort and thus the lower the driver's optimal effort level. Secondly, using $\partial \mathbf{E}\left[u_{i}\left(w_{i}-\tilde{\ell}_{i}, \hat{e}_{i}\right) \mid y\right] / \partial y<0$ gives $\partial \hat{e}_{i} / \partial y>0$ : the larger the expected damages in the case of an accident, the larger the effort. Finally, the driver's risk aversion implies $\partial \hat{e}_{i} / \partial w_{i}<0$ : indeed, the larger the driver's wealth, the lower the expected utility decrease in the case of an accident (because the marginal utility of wealth is decreasing) and thus the lower the incentive to careful driving. These are intuitive behavioral properties and one can easily check that they still hold if $\partial^{2} u_{i} / \partial w_{i}^{f} \partial e_{i} \leq 0$.

This is no more the case when $\partial^{2} u_{i} / \partial w_{i}^{f} \partial e_{i}>0$. For the sake of illustration, let us assume that effort is monetary: $u_{i}\left(w_{i}^{f}, e_{i}\right) \equiv v_{i}\left(w_{i}^{f}-e_{i}\right)$. Consider first the relationship between $x$ and $\hat{e}_{i}$. If $\partial^{2} p / \partial e_{i} \partial x=0$, that is, if $p\left(e_{i}, x\right)$ is separable, then $\partial \hat{e}_{i} / \partial x$ is positive: the safer the network, the larger the prevention expenditures! This counterintuitive 
result goes through a wealth effect. Indeed, for a given $e_{i}$, an increase in $x$ reduces the probability of being in the accident state, hence a decrease in the expected marginal disutility of prevention expenditures-this is the second term in (5). In such a case, the driver would have an incentive to increase the prevention expenditures after an increase in $x$. This wealth effect may dominate if $\partial^{2} p / \partial e_{i} \partial x$ is positive but small: in such a case, an increase in the road safety would induce a larger effort. Likewise, the wealth effect may perturb the relationship between $y$ and $e_{i}$. Indeed, the larger the accident expected costs, the larger the expected marginal disutility of prevention expenditures (because these expenditures have also to be paid in the accident state) and thus the lower the driver's optimal effort. Similarly, an increase in $w_{i}$ decreases the expected marginal disutility of prevention expenditures, hence a larger incentive to spend more money on prevention. Proposition 1 recapitulates this discussion.

Proposition 1: When $\partial^{2} u_{i} / \partial w_{i}^{f} \partial e_{i} \leq 0$, then $\partial \hat{e}_{i} / \partial x<0$ if $\partial^{2} p / \partial e_{i} \partial x>0, \partial \hat{e}_{i} / \partial y>0$, and $\partial \hat{e}_{i} / \partial w_{i}<0$. When $\partial^{2} u_{i} / \partial w_{i}^{f} \partial e_{i}>0$, a wealth effect may invalidate the previous results. Let

$$
\hat{p}_{i}\left(x, y, w_{i}\right)=p\left(\hat{e}_{i}\left(x, y, w_{i}\right), x\right)
$$

be the probability of an accident which follows from the driver's optimal behavior and from the road network parameters $x$ and $y$. When $\partial^{2} u_{i} / \partial w_{i}^{f} \partial e_{i} \leq 0$, and in particular when utility is separable between wealth and effort, then Proposition 1 gives $\partial \hat{p}_{i} / \partial y<0$ and $\partial \hat{p}_{i} / \partial w_{i}>0$ while $\partial \hat{p}_{i} / \partial x$ may be negative or positive. In words, in such a case, an increase in the expected cost of an accident or a decrease in the initial wealth of a risk-averse individual lowers the probability of accident because of a more cautious driving. Conversely, one cannot maintain with certainty that an increase in the $x$ parameter leads to a decrease in the probability of accident, since the beneficial direct effect of safer highways may be offset by the behavioral adaptations of drivers. Results are even more ambiguous when $\partial^{2} u_{i} / \partial w_{i}^{f} \partial e_{i}>0$-which includes the case of a monetary effort-because the sign of the partial derivatives of $\hat{e}_{i}\left(x, y, w_{i}\right)$ cannot be signed with certainty. On the whole, it turns out that the sign of the total effect of $x, y$, and $w_{i}$ on $p_{i}$ cannot be determined on theoretical grounds only: it is a matter of empirical analysis.

\section{Cost-Benefit Analysis of Road Safety Programs}

It is sometimes argued that the behavioral adaptation affecting a road safety policy should be taken into account in appraising its desirability. For instance, according to the OECD expert group: "The potential for behavioral adaptation affecting a safety measure should be considered in estimating the costs and benefits of safety programs. Programs with minimal adaptation may be more effective, in the long run, than those which produce large initial safety gains, but also produce adaptations that eliminate the gain" (OECD, 1990, p. 118). While such a recommendation sounds intuitive and seems to make sense, it lets open the question of how and to what extent the behavioral-adaptation effect should be taken into account in cost-benefit analysis. 
A standard rule would recommend to compare the willingness to pay of road users for a safety measure to its cost. The users' willingness to pay may be derived indirectly by estimating the effects of the measure in terms of human and nonhuman benefits (including the economic value of the decrease in the number of deaths and nonfatal accidents). How should the behavioral-adaptation phenomenon be taken into account in this balance of costs and benefits?

To answer this question, we will insert the analysis of the previous section in a simple one-good model of optimal resource allocation under uncertainty and we will derive simple rules for optimal public decision making. In what follows, we focus on the case where accident costs are borne only by the individuals directly involved in the crashes. We will later extend our results to the more general setting where private costs and social costs may differ because of external accident costs.

\section{A Simple Model of Optimal Resource Allocation}

The insurance indemnity schedule of each driver $i$ is supposed to be given. It determines the probability distribution of $\tilde{\ell}_{i}$ and also the driver $i^{\prime}$ s effort strategy $\hat{e}_{i}\left(x, y, w_{i}\right)$ and his or her probability of accident $\hat{p}_{i}\left(x, y, w_{i}\right)$. Insurers are assumed to offer insurance policies at actuarial fair premium. In case of an accident, the insurance indemnity is $\tilde{y}_{i}-\tilde{\ell}_{i}$. Hence, we have

$$
P_{i}=\hat{p}_{i}\left(x, y, w_{i}\right)\left[y-\mathbf{E}\left(\tilde{\ell}_{i} \mid y\right)\right]
$$

The $x, y$ parameters are affected by road safety programs. These programs entail public expenditures $C(x, y)$ with $\partial C / \partial x>0$ and $\partial C / \partial y<0 .^{7}$

The government budget constraint is written as

$$
\sum_{i=1}^{n} t_{i}=C(x, y)
$$

Using (1), (8), and (9) gives

$$
\sum_{i=1}^{n} w_{i}+\sum_{i=1}^{n} \hat{p}_{i}\left(x, y, w_{i}\right)\left[y-\mathbf{E}\left(\tilde{\ell}_{i} \mid y\right)\right]+C(x, y)=\sum_{i=1}^{n} \bar{w}_{i}
$$

Let $V_{i}\left(w_{i}, x, y\right)$ be the optimal expected utility of driver $i$ with

$$
V_{i}\left(w_{i}, x, y\right)=\max _{e_{i}}\left\{\left[1-p\left(e_{i}, x\right)\right] u_{i}\left(w_{i}, e_{i}\right)+p\left(e_{i}, x\right) \mathbf{E}\left[u_{i}\left(w_{i}-\tilde{\ell}_{i}, e_{i}\right) \mid y\right]\right\}
$$

The government chooses $t_{i}$ (for $\left.i=1, \ldots, n\right), x$, and $y$. Equivalently, the government chooses $w_{i}$ (for $\left.i=1, \ldots, n\right), x$, and $y$. A Pareto improving road safety policy

\footnotetext{
${ }^{7}$ Note that a safety improvement increases $x$ but reduces $y$.
} 
corresponds to (infinitesimal) changes in the road transport system $d x$ and $d y$ and in the drivers' wealth levels $d w_{i}$, for $i=1, \ldots, n$, such that firstly the expected utility of all drivers is nondecreasing and it is increasing for at least one driver and, secondly, the government budget constraint is satisfied. Let us first consider a prevention policy, that is, a policy which affects the $x$ parameter.

The first condition may be written as

$$
\frac{\partial V_{i}}{\partial w_{i}} d w_{i}+\frac{\partial V_{i}}{\partial x} d x \geq 0
$$

for all $i=1, \ldots, n$ with a strict inequality for at least one driver $i$. The second condition is

$$
\sum_{i=1}^{n}\left[1+\frac{\partial \hat{p}_{i}}{\partial w_{i}}\left(y-\mathbf{E}\left(\tilde{\ell}_{i} \mid y\right)\right)\right] d w_{i}+\sum_{i=1}^{n} \frac{\partial \hat{p}_{i}}{\partial x}\left[y-\mathbf{E}\left(\tilde{\ell}_{i} \mid y\right)\right] d x+\frac{\partial C}{\partial x} d x=0
$$

When $d x>0$, there exist compensatory transfers $d w_{i}$, for $i=1, \ldots, n$, such that (12) and (13) are satisfied if and only if

$$
\sum_{i=1}^{n} \frac{\partial V_{i} / \partial x}{\partial V_{i} / w_{i}}\left[1+\frac{\partial \hat{p}_{i}}{\partial w_{i}}\left(y-\mathbf{E}\left(\tilde{\ell}_{i} \mid y\right)\right)\right]-\sum_{i=1}^{n} \frac{\partial \hat{p}_{i}}{\partial x}\left[y-\mathbf{E}\left(\tilde{\ell}_{i} \mid y\right)\right]>\frac{\partial C}{\partial x}
$$

Equation (14) is the condition to be satisfied for an intensification of the prevention policy (i.e., an increase in $x$ ) to be welfare improving.

Likewise, a similar calculation shows that an intensification of the protection policy (i.e., a decrease in $y$ ) is welfare improving if and only if

$$
-\sum_{i=1}^{n} \frac{\partial V_{i} / \partial y}{\partial V_{i} / w_{i}}\left[1+\frac{\partial \hat{p}_{i}}{\partial w_{i}}\left(y-\mathbf{E}\left(\tilde{\ell}_{i} \mid y\right)\right)\right]+\sum_{i=1}^{n} \frac{\partial}{\partial y}\left[\hat{p}_{i}\left(y-\mathbf{E}\left(\tilde{\ell}_{i} \mid y\right)\right)\right]>-\frac{\partial C}{\partial y}
$$

Equations (14) and (15) are cost-benefit decision rules. The left-hand side and the righthand side in (14) and (15) are, respectively, the marginal social value and the marginal cost of an increase in $x$ or of a decrease in $y$. If the marginal social value exceeds the marginal cost, then an increase in $x$ or a decrease in $y$ is welfare improving. More precisely, such policies are potentially Pareto improving in the sense that, under (14) or (15), there exist possible compensatory transfers that make everyone as well off. In what follows, the distribution of tax burdens to pay for government safety projects is not specified, so the condition called (for the sake of brevity) "Pareto improvements" are actually potential Pareto improvement.

Let us consider a prevention policy. Set

$$
W P_{i}^{x}=\frac{\partial V_{i} / \partial x}{\partial V_{i} / \partial w_{i}}
$$


and

$$
\overline{W P}_{i}^{x}=W P_{i}^{x}\left[1+\frac{\partial \hat{p}_{i}}{\partial w_{i}}\left(y-\mathbf{E}\left(\tilde{\ell}_{i} \mid y\right)\right)\right]
$$

$W P_{i}^{x}$ is the marginal willingness to pay of driver $i$ for an increase in $x \cdot \overline{W P}_{i}^{x}$ is an adjusted marginal willingness to pay that takes into account the effect of $w_{i}$ on insurance costs (due to adjustment in the insurance premium under a policy of full risk rating). We will refer to $\overline{W P}_{i}^{x}$ as the adjusted willingness to pay of driver i for the prevention policy.

Let

$$
I_{i}^{x}=-\frac{\partial \hat{p}_{i}}{\partial x}\left[y-\mathbf{E}\left(\tilde{\ell}_{i} \mid y\right)\right]
$$

$I_{i}^{x}$ is the decrease in expected insurance costs which results from an increase in $x . I_{i}^{x}$ is positive under partial behavioral risk compensation, i.e., when $\partial \hat{p}_{i} / \partial x<0$. It would be negative when $\partial \hat{p}_{i} / \partial x>0$, if there were overcompensation of the preventive measure through more risky driving.

Likewise, and with similar interpretations, let

$$
\begin{aligned}
& W P_{i}^{y}=-\frac{\partial V_{i} / \partial y}{\partial V_{i} / w_{i}}, \\
& \overline{W P}_{i}^{y}=W P_{i}^{y}\left[1+\frac{\partial \hat{p}_{i}}{\partial w_{i}}\left(y-\mathbf{E}\left(\tilde{\ell}_{i} \mid y\right)\right)\right],
\end{aligned}
$$

and

$$
I_{i}^{y}=\frac{\partial}{\partial y}\left[\hat{p}_{i}\left(y-\mathbf{E}\left(\tilde{\ell}_{i} \mid y\right)\right)\right]
$$

Hence, rewriting (14) and (15) more compactly, we obtain the following result.

Proposition 2: A preventive road safety measure is Pareto improving if

$$
\sum_{i=1}^{n} \overline{W P}_{i}^{x}+\sum_{i=1}^{n} I_{i}^{x}>\frac{\partial C}{\partial x}
$$

and a protective road safety measure is Pareto improving if

$$
\sum_{i=1}^{n} \overline{W P}_{i}^{y}+\sum_{i=1}^{n} I_{i}^{y}>-\frac{\partial C}{\partial y}
$$

Proposition 2 says that a road safety measure (be it preventive or protective) is Pareto improving if its marginal cost is lower than the corresponding sum of the drivers' 
adjusted marginal willingness to pay for such a measure and of the reduction in insurance costs.

\section{Estimating the Drivers' Willingness to Pay for a Road Safety Measure}

However, it is unlikely that the drivers' (adjusted) marginal willingness to pay for a road safety measure will be easily observed by the Government: this may be considered as an hidden information privately hold by drivers. The conditions in Proposition 2 are thus of little help unless we turn them into equivalent (or approximately equivalent) criteria that do not depend too heavily on such an hidden information. Fortunately, we can connect the driver's marginal willingness to pay for a safety measure to the effects of this measure on losses.

Let us consider first a prevention measure. Using the envelope theorem then gives

$$
W P_{i}^{x}=\frac{\partial p}{\partial x}\left(\hat{e}_{i}, x\right) \frac{u_{i}\left(w_{i}, \hat{e}_{i}\right)-\mathbf{E}\left[u_{i}\left(w_{i}-\tilde{\ell}_{i}, \hat{e}_{i}\right) \mid y\right]}{\left(1-p_{i}\right) \frac{\partial u_{i}\left(w_{i}, \hat{e}_{i}\right)}{\partial w_{i}^{f}}+p_{i} \mathbf{E}\left[\frac{\partial u_{i}\left(w_{i}-\tilde{\ell}_{i}, \hat{e}_{i}\right)}{\partial w_{i}^{f}} \mid y\right]}
$$

where $p_{i} \equiv p\left(\hat{e}_{i}, x\right)$. Using (19) allows us to show that, if the probability of an accident is not too large then the marginal willingness to pay of a risk-averse driver for an increase in $x$ is larger than the corresponding decrease in his expected uninsured damages with unchanged behavior. This is established in the following lemma. ${ }^{8}$

Lemma 1: Assume $M_{i}<\frac{\partial^{2} u_{i}\left(w, \hat{e}_{i}\right)}{\partial w^{2}}<m_{i}<0$ for all $w$. Then

$$
\frac{\partial V_{i} / \partial x}{\partial V_{i} / \partial w_{i}}>-\frac{\partial p}{\partial x}\left(\hat{e}_{i}, x\right) \mathbf{E}\left(\tilde{\ell}_{i} \mid y\right) \quad \text { if } 0 \leq p_{i} \leq \frac{m_{i}}{M_{i}+m_{i}}
$$

Proof: See the Appendix.

Lemma 1 can be interpreted as follows. Assume that the effort of driver $i$ is fixed at its optimal level $e_{i}=\hat{e}_{i}$ and consider a small variation $d x$. Let

$$
d t_{i}^{*}=-\frac{\partial p}{\partial x}\left(\hat{e}_{i}, x\right) \mathbf{E}\left(\tilde{\ell}_{i} \mid y\right) d x
$$

be a transfer from driver $i$ to the government which compensates for the variation in the accident probability $d p_{i}=\left[\partial p\left(\hat{e}_{i}, x\right) / \partial x\right] d x$ in such a way that driver $i$ 's expected wealth is kept constant. Hence $\left(d x, d t_{i}^{*}\right)$ induces a mean preserving variation in driver $i$ 's wealth. Assume $d x<0$, which gives $d p_{i}>0$. Such a change puts more weight on the "accident" state and less weight on the "no-accident" state. It is in fact a mean preserving spread in the sense of Rothschild and Stiglitz (1970) if the probability of the "accident" state is not too large. Otherwise, it would be a mean preserving

\footnotetext{
${ }^{8}$ Note that more restrictive conditions on $u_{i}($.$) would allow to relax the upper bound on p_{i}$ under which the inequality of Lemma 1 holds. In particular, if $u_{i}($.$) exhibits mixed risk aversion$ in the sense of Caballé and Pomansky (1996), then as shown by Dachraoui et al. (2000), the inequality of Lemma 1 holds if $p_{i}<1 / 2$.
} 
contraction. Driver $i$ is negatively affected by a mean preserving spread in his (or her) wealth because he (or she) is risk averse. Hence, if $p_{i}$ is not too large, the net transfer to driver $i$ denoted by $d w_{i}$ which would exactly compensate for the utility loss induced by the increase in the accident probability is such that $d w_{i}>-d t_{i}^{*}$, which implies

$$
\frac{d w_{i}}{d x}>-\frac{\partial p}{\partial x}\left(\hat{e}_{i}, x\right) \mathbf{E}\left(\tilde{\ell}_{i} \mid y\right)
$$

This gives a lower bound for the marginal willingness to pay for an increase in $x$, when $\hat{e}_{i}$ is fixed at its optimal level. Because of the envelope theorem, the fact that driver $i$ optimally modifies his or her effort level in response to changes in $x$ does not change the result.

Using (19) also allows us to get an estimate of the marginal willingness to pay for a prevention measure when $p_{i}$ is small. Indeed, we have

$$
\lim _{p_{i \rightarrow 0}} W P_{i}^{x} \times \frac{1}{\frac{\partial p}{\partial x}\left(\hat{e}_{i}, x\right)}=\frac{u_{i}\left(w_{i}, \hat{e}_{i}\right)-\mathbf{E}\left[u_{i}\left(w_{i}-\tilde{\ell}_{i}, \hat{e}_{i}\right) \mid y\right]}{\frac{\partial u_{i}\left(w_{i}, \hat{e}_{i}\right)}{\partial w_{i}^{f}}}
$$

Using a second-order Taylor expansion gives

$$
\mathbf{E}\left[u_{i}\left(w_{i}-\tilde{\ell}_{i}, \hat{e}_{i}\right) \mid y\right] \simeq u_{i}\left(w_{i}, \hat{e}_{i}\right)-\frac{\partial u_{i}\left(w_{i}, \hat{e}_{i}\right)}{\partial w_{i}^{f}} \mathbf{E}\left(\tilde{\ell}_{i} \mid y\right)+\frac{1}{2} \frac{\partial^{2} u_{i}\left(w_{i}, \hat{e}_{i}\right)}{\partial w_{i}^{f 2}} \mathbf{E}\left(\tilde{\ell}_{i}^{2} \mid y\right)
$$

Equations (20) and (21) then yield

$$
\lim _{p_{i \rightarrow 0}} W P_{i}^{x} \times \frac{1}{\frac{\partial p}{\partial x}\left(\hat{e}_{i}, x\right)} \simeq \mathbf{E}\left(\tilde{\ell}_{i} \mid y\right)\left[1+\frac{R_{i}\left(w_{i}, \hat{e}_{i}\right) \mathbf{E}\left(\tilde{\ell}_{i}^{2} \mid y\right)}{2 w_{i} \mathbf{E}\left(\tilde{\ell}_{i} \mid y\right)}\right]
$$

where $R_{i}\left(w, \hat{e}_{i}\right) \equiv-w \frac{\partial^{2} u_{i}\left(w, \hat{e}_{i}\right)}{\partial w^{2}} / \frac{\partial u_{i}\left(w, \hat{e}_{i}\right)}{\partial w}$ is the coefficient of relative risk aversion.

As an example, assume $\tilde{\ell}_{i} \rightsquigarrow \exp (\lambda)$ with $\lambda^{-1}=\mathbf{E}\left(\tilde{\ell}_{i} \mid y\right)$. We then have $\mathbf{E}\left(\tilde{\ell}_{i}^{2} \mid y\right) /$ $\mathbf{E}\left(\tilde{\ell}_{i} \mid y\right)=2 \mathbf{E}\left(\tilde{\ell}_{i} \mid y\right)$. Assume furthermore $\lambda^{-1}=\$ 1000, w_{i}=\$ 25,000$, and $R_{i}\left(w_{i}, \hat{e}_{i}\right) \in$ $[1,4] .{ }^{9}$ We then have

$$
\frac{R_{i}\left(w_{i}, \hat{e}_{i}\right) \mathbf{E}\left(\tilde{\ell}_{i}^{2} \mid y\right)}{2 w_{i} \mathbf{E}\left(\tilde{\ell}_{i} \mid y\right)} \in[0.04,0.16]
$$

which suggests that, in practice, the difference

$$
W P_{i}^{x}-\frac{\partial p}{\partial x}\left(\hat{e}_{i}, x\right) \mathbf{E}\left(\tilde{\ell}_{i} \mid y\right)
$$

is probably small for reasonable estimates of the coefficient of relative risk aversion.

\footnotetext{
${ }^{9}$ See Gollier (2001, p. 31) on why a coefficient of relative risk aversion between 1 and 4 is a reasonable assumption.
} 
Hence we may write

$$
W P_{i}^{x}=-\theta_{i}^{1} \frac{\partial p}{\partial x}\left(\hat{e}_{i}, x\right) \mathbf{E}\left(\tilde{\ell}_{i} \mid y\right)
$$

with $\theta_{i}^{1}>1$ and with reasons to think that, on real data, the difference between $\theta_{i}^{1}$ and 1 is probably not very large. ${ }^{10}$

Likewise, using the envelope theorem gives

$$
-\frac{\partial V_{i} / \partial y}{\partial V_{i} / \partial w_{i}}=p_{i} \frac{\frac{\partial}{\partial y} \mathbf{E}\left[u_{i}\left(w_{i}-\tilde{\ell}_{i}, \hat{e}_{i}\right) \mid y\right]}{\left(1-p_{i}\right) \frac{\partial u_{i}\left(w_{i}, \hat{e}_{i}\right)}{\partial w_{i}^{f}}+p_{i} \mathbf{E}\left[\frac{\partial u_{i}\left(w_{i}-\tilde{\ell}_{i}, \hat{e}_{i}\right)}{\partial w_{i}^{f}} \mid y\right]}
$$

where $p_{i}=p\left(\hat{e}_{i}, x\right)$.

Lemma 2: Assume that $\tilde{\ell}_{i}$ is distributed on $[0, \bar{y}], \bar{y}>0$, according to a density function $f\left(\tilde{\ell}_{i} \mid y\right)$. Let $F\left(\tilde{\ell}_{i} \mid y\right)$ denote the corresponding cumulative distribution function. Assume that $u_{i}^{\prime \prime}<0$ and that $\frac{\frac{\partial F}{\partial y}\left(\tilde{\ell}_{i} \mid y\right)}{f\left(\tilde{\ell}_{i} \mid y\right)}$ is nonincreasing with respect to $\tilde{\ell}_{i}$. Then

$$
-\frac{\partial V_{i} / \partial y}{\partial V_{i} / \partial w_{i}}>p_{i} \frac{\partial \mathbf{E}\left(\tilde{\ell}_{i} \mid y\right)}{\partial y}
$$

Proof: See the Appendix.

Lemma 2 provides a condition under which the willingness to pay of a risk-averse driver for an intensification in the protection policy (i.e., for a decrease in $y$ ) is larger than the corresponding decrease in expected uninsured accident costs for an unchanged driving effort level. The intuitive meaning of this condition is as follows. Let us remind that an increase in $y$ shifts the distributions of $\tilde{y}$ and $\tilde{\ell}_{i}$ in the sense of first-order stochastic dominance. Consider a decrease in $y$, which gives a decrease in the average cost of accidents. Because the marginal utility of wealth is decreasing, the willingness to pay of a risk-averse driver for such a protection policy will be higher when this policy lowers the probability of the most serious accidents than when it reduces the gravity of minor accidents. In Lemma 2, we make an assumption to express the fact that the protection policy affects more intensely the most serious accidents (i.e., the accidents with large damages, particularly fatalities) than the accidents with slight material damages. In mathematical terms, we postulate that, following an increase in $y$, the rightward shift of the cumulative distribution $F\left(\tilde{\ell}_{i} \mid y\right)$-if we measure it horizontally-is nondecreasing with $\tilde{\ell}_{i}$. This is true if $\frac{\partial F}{\partial y}\left(\tilde{\ell}_{i} \mid y\right) / f\left(\tilde{\ell}_{i} \mid y\right)$ is nonincreasing in $\tilde{\ell}_{i}$ which is assumed in what follows.

As an illustration, we may get an estimate of the marginal willingness to pay for a protection measure when $p_{i}$ is small and $\tilde{\ell}_{i} \rightsquigarrow \exp (\lambda)$ with $\lambda^{-1}=\mathrm{E}\left(\tilde{\ell}_{i} \mid y\right)$. We have

${ }^{10}$ Note that $\theta_{i}^{1}$ would be equal to 1 if driver $i$ were risk neutral. 


$$
\lim _{p_{i \rightarrow 0}} W P_{i}^{y} \times \frac{1}{p_{i}}=\frac{\frac{\partial}{\partial y} \mathbf{E}\left[u_{i}\left(w_{i}-\tilde{\ell}_{i}, \hat{e}_{i}\right) \mid y\right]}{\frac{\partial u_{i}\left(w_{i}, \hat{e}_{i}\right)}{\partial w_{i}^{f}}}
$$

and a simple calculation gives

$$
\frac{\partial}{\partial y} \mathbf{E}\left[u_{i}\left(w_{i}-\tilde{\ell}_{i}, \hat{\ell}_{i}\right) \mid y\right]=\frac{\frac{\partial \mathbf{E}\left(\tilde{\ell}_{i} \mid y\right)}{\partial y}}{\mathbf{E}\left(\tilde{\ell}_{i} \mid y\right)} \int_{0}^{+\infty} \frac{\partial u_{i}\left(w_{i}-\tilde{\ell}_{i}, \hat{e}_{i}\right)}{\partial w_{i}^{f}} \tilde{\ell}_{i} f\left(\tilde{\ell}_{i} \mid y\right) d \tilde{\ell}_{i}
$$

Using a second-order Taylor expansion of $\partial u_{i}\left(w_{i}-\tilde{\ell}_{i}, \hat{e}_{i}\right) / \partial w_{i}^{f}$ around $\partial u_{i}\left(w_{i}, \hat{e}_{i}\right) / \partial w_{i}^{f}$ then yields

$$
\frac{\frac{\partial}{\partial y} \mathbf{E}\left[u_{i}\left(w_{i}-\tilde{\ell}_{i}, \hat{e}_{i}\right) \mid y\right]}{\frac{\partial u_{i}\left(w_{i}, \hat{e}_{i}\right)}{\partial w_{i}^{f}}} \simeq \frac{\partial \mathbf{E}\left(\tilde{\ell}_{i} \mid y\right)}{\partial y}\left[1+\frac{R_{i}\left(w_{i}, \hat{e}_{i}\right) \mathbf{E}\left(\tilde{\ell}_{i} \mid y\right)}{w_{i}}\right]
$$

Hence, for instance, when $\lambda^{-1}=\$ 1000, w_{i}=\$ 25,000$ and $R_{i}\left(w_{i}\right) \in[1,4]$, we get

$$
1.04 \times p_{i} \frac{\partial \mathbf{E}\left(\tilde{\ell}_{i} \mid y\right)}{\partial y} \leq W P_{i}^{y} \leq 1.16 \times p_{i} \frac{\partial \mathbf{E}\left(\tilde{\ell}_{i} \mid y\right)}{\partial y}
$$

Of course, we should not give too much importance to such an example but once again it suggests that the adjustment of the marginal willingness to pay due to risk aversion is probably small.

Hence we may write

$$
W P_{i}^{y}=\theta_{i}^{2} p_{i} \frac{\partial \mathbf{E}\left(\tilde{\ell}_{i} \mid y\right)}{\partial y}
$$

with $\theta_{i}^{2}>1 .{ }^{11}$ These results are summarized in the following Proposition.

Proposition 3: Under the assumptions made in Lemmas 1 and 2, we have

$$
\begin{gathered}
W P_{i}^{x}=-\theta_{i}^{1} \frac{\partial p}{\partial x}\left(\hat{e}_{i}, x\right) \mathbf{E}\left(\tilde{\ell}_{i} \mid y\right), \\
W P_{i}^{y}=\theta_{i}^{2} p_{i} \frac{\partial \mathbf{E}\left(\tilde{\ell}_{i} \mid y\right)}{\partial y}
\end{gathered}
$$

with $\theta_{i}^{1}>1$ and $\theta_{i}^{2}>1$.

${ }^{11}$ Note that $\theta_{i}^{2}$ would be equal to one if driver $i$ were risk neutral. 


\section{Cost-Benefit Criteria}

Proposition 3 allows us to rewrite the total marginal benefits of road safety measures in a more explicit way. Using (27) gives

$$
\begin{aligned}
\sum_{i=1}^{n} \overline{W P}_{i}^{x}+\sum_{i=1}^{n} I_{i}^{x}= & -\sum_{i=1}^{n} \theta_{i}^{1} \frac{\partial p}{\partial x}\left(\hat{e}_{i}, x\right) \mathbf{E}\left(\tilde{\ell}_{i} \mid y\right)\left[1+\frac{\partial \hat{p}_{i}}{\partial w_{i}}\left(y-\mathbf{E}\left(\tilde{\ell}_{i} \mid y\right)\right)\right] \\
& -\sum_{i=1}^{n} \frac{\partial \hat{p}_{i}}{\partial x}\left[y-\mathbf{E}\left(\tilde{\ell}_{i} \mid y\right)\right] \\
= & \sum_{i=1}^{n}\left[y+\left(\theta_{i}^{1}-1\right) \mathbf{E}\left(\tilde{\ell}_{i} \mid y\right)\right] \frac{\partial p}{\partial x}\left(\hat{e}_{i}, x\right) \\
& -\sum_{i=1}^{n} \frac{\partial p}{\partial e}\left(\hat{e}_{i}, x\right)\left[\frac{\partial \hat{e}_{i}}{\partial x}(x, y, w)+\theta_{i}^{1} \frac{\partial p}{\partial x}\left(\hat{e}_{i}, x\right) \mathbf{E}\left(\tilde{\ell}_{i} \mid y\right) \frac{\partial \hat{e}_{i}}{\partial w_{i}}(x, y, w)\right] \\
& \times\left[y-\mathbf{E}\left(\tilde{\ell}_{i} \mid y\right)\right]
\end{aligned}
$$

and using (28) gives

$$
\begin{aligned}
\sum_{i=1}^{n} \overline{W P}_{i}^{y}+\sum_{i=1}^{n} I_{i}^{y}= & \sum_{i=1}^{n}\left[\theta_{i}^{2} p_{i} \frac{\partial \mathbf{E}\left(\tilde{\ell}_{i} \mid y\right)}{\partial y}\right]\left[1+\frac{\partial \hat{p}_{i}}{\partial w_{i}}\left(y-\mathbf{E}\left(\tilde{\ell}_{i} \mid y\right)\right)\right] \\
& +\sum_{i=1}^{n} p_{i}\left[1-\frac{\partial \mathbf{E}\left(\tilde{\ell}_{i} \mid y\right)}{\partial y}\right]+\sum_{i=1}^{n} \frac{\partial}{\partial y}\left[\hat{p}_{i}\left(y-\mathbf{E}\left(\tilde{\ell}_{i} \mid y\right)\right)\right] \\
= & \sum_{i=1}^{n}\left[1+\left(\theta_{i}^{2}-1\right) \frac{\partial \mathbf{E}\left(\tilde{\ell}_{i} \mid y\right)}{\partial y}\right] p\left(e_{i}, x\right) \\
& -\sum_{i=1}^{n} \frac{\partial p}{\partial e}\left(\hat{e}_{i}, x\right)\left[\frac{\partial \hat{e}_{i}}{\partial y}(x, y, w)+\theta_{i}^{2} p_{i} \frac{\partial \mathbf{E}\left(\tilde{\ell}_{i} \mid y\right)}{\partial y} \frac{\partial \hat{e}_{i}}{\partial w_{i}}(x, y, w)\right] \\
& \times\left[y-\mathbf{E}\left(\tilde{\ell}_{i} \mid y\right)\right] .
\end{aligned}
$$

Hence the following Proposition.

Proposition 4: A preventive road safety measure is Pareto improving if and only if

$$
-\sum_{i=1}^{n}\left[y+\left(\theta_{i}^{1}-1\right) \mathbf{E}\left(\tilde{\ell}_{i} \mid y\right)\right] \frac{\partial p}{\partial x}\left(\hat{e}_{i}, x\right)-\sum_{i=1}^{n} \Delta_{i}^{x} \frac{\partial p}{\partial e}\left(\hat{e}_{i}, x\right)\left[y-\mathbf{E}\left(\tilde{\ell}_{i} \mid y\right)\right]>\frac{\partial C}{\partial x},
$$

where

$$
\Delta_{i}^{x}=\frac{\partial \hat{e}_{i}}{\partial x}(x, y, w)+\theta_{i}^{1} \frac{\partial p}{\partial x}\left(\hat{e}_{i}, x\right) \mathbf{E}\left(\tilde{\ell}_{i} \mid y\right) \frac{\partial \hat{e}_{i}}{\partial w_{i}}(x, y, w)
$$


A protective road safety measure is Pareto improving if and only if

$$
\sum_{i=1}^{n} p_{i}\left[1+\left(\theta_{i}^{2}-1\right) \frac{\partial \mathbf{E}\left(\tilde{\ell}_{i} \mid y\right)}{\partial y}\right]+\sum_{i=1}^{n} \Delta_{i}^{y} \frac{\partial p}{\partial e}\left(\hat{e}_{i}, x\right)\left[y-\mathbf{E}\left(\tilde{\ell}_{i} \mid y\right)\right]>-\frac{\partial C}{\partial y}
$$

where

$$
\Delta_{i}^{y}=\frac{\partial \hat{e}_{i}}{\partial y}(x, y, w)+\theta_{i}^{2} p_{i} \frac{\partial \mathbf{E}\left(\tilde{\ell}_{i} \mid y\right)}{\partial y} \frac{\partial \hat{e}_{i}}{\partial w_{i}}(x, y, w)
$$

The first term in the left-hand side of (31) is the direct marginal welfare effect of a prevention measure with unchanged behaviors. For each driver, this direct effect is the sum of the decrease in the total losses which results from a unit increase in $x$ with unchanged behaviors (i.e., $-y \partial p / \partial x)$ and of an adjustment term $-\left(\theta_{i}^{1}-1\right) \mathbf{E}\left(\tilde{\ell}_{i} \mid y\right) \partial p / \partial x$ that gives a positive social value to the decrease in uninsured losses incurred by riskaverse drivers. ${ }^{12}$ This first term may be called the adjusted direct welfare gain of the prevention measure. The second term in the left-hand side of (31) is the increase in insured losses following a unit increase in $x$, because of drivers' behavioral adaptation. Indeed, observe that $\Delta_{i}^{x} d x$ is the effort variation resulting from an infinitesimal variation $d x$. This effort variation includes the direct effect $\partial \hat{e}_{i} / \partial x$ and the (adjusted for risk aversion) wealth effect $\theta_{i}^{1} \mathbf{E}\left(\tilde{\ell}_{i} \mid y\right)\left[\partial p\left(\hat{e}_{i}, x\right) / \partial x\right] \partial \hat{e}_{i} / \partial w_{i}$ due to the change in the uninsured losses. Multiplying this effort variation $\Delta_{i}^{x}$ by $\left[\partial p\left(\hat{e}_{i}, x\right) / \partial e\right]\left[y-\mathbf{E}\left(\tilde{\ell}_{i} \mid y\right)\right]$ gives the effect of behavioral adaptation on insured losses. In a word, the first part of Proposition 4 says that a more intensive prevention policy (associated with adequately chosen compensatory transfers) is Pareto improving when its marginal cost is lower than the difference between the adjusted direct welfare gain and the effect of behavioral adaptation on insured losses.

Note that a conventional cost-benefit study would usually identify the benefits of a prevention measure with the first term only, probably ignoring the adjustment for risk aversion. This is usually done by estimating the decrease in human or nonhuman losses that would have been possible in given circumstances (particularly with given driving behaviors) if the prevention measure had been previously adopted. Extrapolating the results from these case studies to the whole road transport system would allow the public decision maker to appraise the desirability of a prevention measure. Our results show that the additional expected insured losses resulting from behavioral adaptation should be deduced from the previous estimate of net benefits of a traffic safety policy.

The second part of Proposition 4 can be rephrased in similar terms. Indeed $\left\{p_{i}[1+\right.$ $\left.\left.\left(\theta_{i}^{2}-1\right) \partial \mathbf{E}\left(\tilde{\ell}_{i} \mid y\right) / \partial y\right]\right\} d y$ is the adjusted direct welfare gain of the protection measure $d y$ for driver $i$ and $\Delta_{i}^{y}\left[\partial p\left(\hat{e}_{i}, x\right) / \partial e\right]\left[y-\mathbf{E}\left(\tilde{\ell}_{i} \mid y\right)\right] d y$ is the effect of behavioral adaptation on insured losses with $\Delta_{i}^{y} d y$ the induced effort variation. Hence, Proposition 4 says that a more intensive protection policy is Pareto improving if its marginal cost is less than the difference between the adjusted welfare gain and the effect of behavioral adaptation on insured losses.

\footnotetext{
${ }^{12}$ Note that the adjustment term would vanish if driver $i$ were risk neutral.
} 


\section{Social Cost of Accidents}

This section extends our results to the more general case where accidents may entail some social costs that are not directly incurred by the individuals involved in the car crashes. Individuals only care about the residual value (net of insurance) of the accident private costs but, as regards public policies, what really matters are the social costs of accidents. Private costs and social costs differ especially when the private value of life differs from the social value. The private value of a statistical life measures how much individuals are willing to pay to avoid risks that will result in one less death ${ }^{13}$ but it may differ from the social value of life, for example, when wages differ from marginal productivity of labor or when the premature death of a skilled worker entails a negative external effect in the productive sector. Furthermore, in addition to the monetary and nonmonetary private costs (including pain and suffering) incurred by the individuals who are directly concerned by car accidents, the social costs of motor vehicle crashes also include additional components such as the cost of emergency services, the additional time travel costs for other motorists, the workplace disruptions, and the cost of legal proceedings. ${ }^{14}$ These additional costs are part and parcel of the social costs of accidents.

For the sake of notational simplicity, we will assume that social expected costs and private expected costs are proportional. The total expected social cost of an accident is now written as $(1+\lambda) y$, where $y$ and $\lambda y$, respectively, denote the expected cost incurred by the individuals involved in the accident and the other expected costs. We will refer to $y$ and $\lambda y$ as the expected direct cost and the expected external cost of an accident. Hence, it is assumed that a protection measure simultaneously decreases these two components.

In what follows, $w_{i}$ and $t_{i}$ have unchanged meanings: $w_{i}$ still denotes the wealth of driver $i$ in the no-accident state and $t_{i}$ denotes the transfer to the government. Since the government can make lump sum transfers to the individuals, it is not restrictive to assume that the external costs of accidents are fully borne by the government budget, either directly in case of monetary expenditures or indirectly through compensatory transfers paid to the affected individuals. With this assumption, Equation (1) still holds but the government budget constraint is now written as

$$
\sum_{i=1}^{n} t_{i}=C(x, y)+\lambda y \sum_{i=1}^{n} \hat{p}_{i}\left(x, y, w_{i}\right)
$$

instead of (9). In words, the transfers to the government should cover at the same time the cost of road safety public expenditures and the external costs of accidents. Consequently, (10) is replaced by

${ }^{13}$ The private value of life may be estimated in several ways, including the wage premia for risky jobs and revealed preferences approaches such as the consumers' willingness to pay for safety features (e.g., safer cars); see Boardman et al. (2001, Ch. 15) for an introduction to the estimation techniques and how the value of life is used in cost-benefit analysis and Viscusi (1993) for an extensive review of both the conceptual issues and the empirical studies on the value of life.

${ }^{14}$ Miller (1993) estimates the cost of US motor vehicle crashes. He points out that not using comprehensive costs measures can significantly distort the cost-benefit analysis of road safety measures. 


$$
\sum_{i=1}^{n} w_{i}+\sum_{i=1}^{n} \hat{p}_{i}\left(x, y, w_{i}\right)\left[(1+\lambda) y-\mathbf{E}\left(\tilde{\ell}_{i} \mid y\right)\right]+C(x, y)=\sum_{i=1}^{n} \bar{w}_{i}
$$

A straightforward adaptation of the derivation performed in the previous sections then leads to the following Proposition.

Proposition 5: When accidents entail external costs, a preventive road safety measure is Pareto improving if and only if

$$
-\sum_{i=1}^{n}\left[(1+\lambda) y+\left(\theta_{i}^{1}-1\right) \mathbf{E}\left(\tilde{\ell}_{i} \mid y\right)\right] \frac{\partial p}{\partial x}\left(\hat{e}_{i}, x\right)-\sum_{i=1}^{n} \Delta_{i}^{x} \frac{\partial p}{\partial e}\left(\hat{e}_{i}, x\right)\left[(1+\lambda) y-\mathbf{E}\left(\tilde{\ell}_{i} \mid y\right)\right]>\frac{\partial C}{\partial x}
$$

where $\Delta_{i}^{x}$ is given by (32). Similarly, a protective road safety measure is Pareto improving if and only if

$$
\sum_{i=1}^{n} p_{i}\left[1+\lambda+\left(\theta_{i}^{2}-1\right) \frac{\partial \mathbf{E}\left(\tilde{\ell}_{i} \mid y\right)}{\partial y}\right]+\sum_{i=1}^{n} \Delta_{i}^{y} \frac{\partial p}{\partial e}\left(\hat{e}_{i}, x\right)\left[(1+\lambda) y-\mathbf{E}\left(\tilde{\ell}_{i} \mid y\right)\right]>-\frac{\partial C}{\partial y}
$$

where $\Delta_{i}^{y}$ is given by (34).

Proposition 5 extends the results of Proposition 4 to the case where the social costs of accidents may differ from the private costs. The first term in the left-hand side of (37) is the direct monetarized welfare gain induced by the prevention measure: it is the increase in welfare (external effects included) due to the decrease in the accident probability for unchanged behaviors and after adjustment for risk aversion. Proposition 5 shows that the social cost of behavioral adaptation should be deduced from this adjusted direct welfare gain in order to compute the net social benefit of the prevention measure. This social cost of behavioral adaptation now includes the additional insured losses and also the decrease in the external costs of accidents induced by the change in the drivers' behavior. In other words, the external cost of accidents increases at the same time the direct welfare gain of the prevention measure and the social cost of behavioral adaptation. Equation (38) can be interpreted similarly in the case of a protection measure.

\section{CONCLUSION}

It is often argued that ignoring the behavioral adaptation of drivers would bias the cost-benefit analysis of a road safety measure toward positive net benefits of an active traffic safety regulation. However, the way in which behavioral adaptations should be taken into account in the balance of costs and benefits is not self-evident. The purpose of this article was to clarify this issue of how behavioral adaptation should affect conventional cost-benefit analysis in the framework of a simple model of resource allocation under uncertainty. In the end, we got a rather simple result: the net benefits of a road safety policy are written as the difference between the (adjusted for risk aversion) reduction of total losses under unchanged behaviors and the increase in insured losses attributable to behavioral adaptation. Furthermore, the same kind of 
formula applies to prevention measures as to protection policies and they can be adapted so that the external costs of accidents are taken into account.

Such a cost-benefit criterion allows the policymaker to appraise the desirability of additional public investments for highways, but it can also be used for new mandatory automobile safety devices, be they preventive (e.g., antilocking systems) or protective (e.g., seat belts, crash tests,...). Among other possible extensions, we may also note that, in practice, behavioral adaptation to road safety policy also goes through an increase in traffic density, which may induce externalities in the form of congestion costs or of an increase in average accident risk. Furthermore, accidents may also be costly for the drivers not at fault, particularly in case of bodily injuries. Although the main objectives of this article were of a theoretical nature, taking these effects into account would put our analysis back in a more realistic framework. This would certainly be essential for the purpose of implementation on real data.

Note finally that the results derived in this article suggest that there exists some kind of substitutability between private protection from accident risks through insurance and public protection through tax financed expenditures or mandatory safety devices. When agents are more completely insured against accident losses, the social cost of behavioral adaptation is higher and, in such a case, the social value of a more active safety regulation is lower.

\section{ApPendiX}

Proof of Lemma 1: For $\ell_{i}$ fixed, the Taylor inequalities give

$$
\left\{\begin{array}{l}
u_{i}\left(w_{i}, \hat{e}_{i}\right)-u_{i}\left(w_{i}-\ell_{i}, \hat{e}_{i}\right) \geq \ell_{i} \frac{\partial u_{i}\left(w_{i}, \hat{e}_{i}\right)}{\partial w_{i}^{f}}-\frac{\ell_{i}^{2}}{2} m_{i} \\
u_{i}\left(w_{i}, \hat{e}_{i}\right)-u_{i}\left(w_{i}-\ell_{i}, \hat{e}_{i}\right) \geq \ell_{i} \frac{\partial u_{i}\left(w_{i}-\ell_{i}, \hat{e}_{i}\right)}{\partial w_{i}^{f}}+\frac{\ell_{i}^{2}}{2} M_{i} .
\end{array}\right.
$$

Hence

$$
\begin{aligned}
u_{i}\left(w_{i}, \hat{e}_{i}\right)-u_{i}\left(w_{i}-\ell_{i}, \hat{e}_{i}\right) \geq \ell_{i} & {\left[\left(1-p_{i}\right) \frac{\partial u_{i}\left(w_{i}, \hat{e}_{i}\right)}{\partial w_{i}^{f}}+p_{i} \frac{\partial u_{i}\left(w_{i}-\ell_{i}, \hat{e}_{i}\right)}{\partial w_{i}^{f}}\right] } \\
& +\frac{\ell_{i}^{2}}{2}\left[p_{i} M_{i}-\left(1-p_{i}\right) m_{i}\right] \\
\geq & \ell_{i}\left[\left(1-p_{i}\right) \frac{\partial u_{i}\left(w_{i}, \hat{e}_{i}\right)}{\partial w_{i}^{f}}+p_{i} \frac{\partial u_{i}\left(w_{i}-\ell_{i}, \hat{e}_{i}\right)}{\partial w_{i}^{f}}\right] .
\end{aligned}
$$

Since $\partial u_{i}\left(w_{i}-\ell_{i}, \hat{e}_{i}\right) / \partial w_{i}^{f}$ is increasing in $\ell_{i}$, both random variables $\tilde{\ell}_{i}$ and $\partial u_{i}\left(w_{i}-\right.$ $\left.\tilde{\ell}_{i}, \hat{e}_{i}\right) / \partial w_{i}^{f}$ are positively correlated. Therefore, we may write

$$
\mathbf{E}\left[\tilde{\ell}_{i} \frac{\partial u_{i}\left(w_{i}-\tilde{\ell}_{i}, \hat{e}_{i}\right)}{\partial w_{i}^{f}} \mid y\right] \geq \mathbf{E}\left[\tilde{\ell}_{i} \mid y\right] \mathbf{E}\left[\frac{\partial u_{i}\left(w_{i}-\tilde{\ell}_{i}, \hat{e}_{i}\right)}{\partial w_{i}^{f}} \mid y\right]
$$


and

$u_{i}\left(w_{i}, \hat{e}_{i}\right)-\mathbf{E}\left[u_{i}\left(w_{i}-\tilde{\ell}_{i}, \hat{e}_{i}\right) \mid y\right] \geq \mathbf{E}\left[\tilde{\ell}_{i} \mid y\right] \mathbf{E}\left[(1-p) \frac{\partial u_{i}\left(w_{i}, \hat{e}_{i}\right)}{\partial w_{i}^{f}}+p \frac{\partial u_{i}\left(w_{i}-\tilde{\ell}_{i}, \hat{e}_{i}\right)}{\partial w_{i}^{f}} \mid y\right]$

Finally, (19) gives the result.

Q.E.D.

Proof of Lemma 2: We have

$$
\mathbf{E}\left[u_{i}\left(w_{i}-\tilde{\ell}_{i}, \hat{e}_{i}\right) \mid y\right]=\int_{0}^{\bar{y}} u_{i}\left(w_{i}-\tilde{\ell}_{i}, \hat{e}_{i}\right) f\left(\tilde{\ell}_{i} \mid y\right) d \tilde{\ell}_{i} .
$$

Integrating by parts gives

$$
\mathbf{E}\left[u_{i}\left(w_{i}-\tilde{\ell}_{i}, \hat{e}_{i}\right) \mid y\right]=u_{i}\left(w_{i}-\bar{y}, \hat{e}_{i}\right)-\int_{0}^{\bar{y}} \frac{\partial u_{i}\left(w_{i}-\tilde{\ell}_{i}, \hat{e}_{i}\right)}{\partial w_{i}^{f}} F\left(\tilde{\ell}_{i} \mid y\right) d \tilde{\ell}_{i} .
$$

We may write

$$
\frac{\partial \mathbf{E}\left[u_{i}\left(w_{i}-\tilde{\ell}_{i}, \hat{\ell}_{i}\right) \mid y\right]}{\partial y}=-\int_{0}^{\bar{y}} \frac{\partial u_{i}\left(w_{i}-\tilde{\ell}_{i}, \hat{\ell}_{i}\right)}{\partial w_{i}^{f}} \frac{\partial F\left(\tilde{\ell}_{i} \mid y\right)}{\partial y} d \tilde{\ell}_{i}
$$

Assuming that $\frac{(\partial F / \partial y)\left(\tilde{\ell}_{i} \mid y\right)}{f\left(\tilde{\ell}_{i} \mid y\right)}$ is nonincreasing in $\tilde{\ell}_{i}$, we have

$$
\frac{\partial \mathbf{E}\left[u_{i}\left(w_{i}-\tilde{\ell}_{i}, \hat{e}_{i}\right) \mid y\right]}{\partial y} \geq-\int_{0}^{\bar{y}} \frac{\partial u_{i}\left(w_{i}-\tilde{\ell}_{i}, \hat{e}_{i}\right)}{\partial w_{i}^{f}} f\left(\tilde{\ell}_{i} \mid y\right) d \tilde{\ell}_{i} \int_{0}^{\bar{y}} \frac{\partial F\left(\tilde{\ell}_{i} \mid y\right)}{\partial y} d \tilde{\ell}_{i}
$$

because $\frac{\partial u_{i}\left(w_{i}-\tilde{\ell}_{i}, \hat{e}_{i}\right)}{\partial w_{i}^{f}}$ and $\frac{(\partial F / \partial y)\left(\tilde{\ell}_{i} \mid y\right)}{f\left(\tilde{\ell}_{i} \mid y\right)}$ are positively correlated random variables. Hence we have

$$
\frac{\partial \mathbf{E}\left[u_{i}\left(w_{i}-\tilde{\ell}_{i}\right) \mid y\right]}{\partial y} \geq \mathbf{E}\left[\frac{\partial u_{i}\left(w_{i}-\tilde{\ell}_{i}, \hat{e}_{i}\right)}{\partial w_{i}^{f}} \mid y\right] \frac{\partial}{\partial y} \mathbf{E}\left(\tilde{\ell}_{i} \mid y\right)
$$

Using (24) then gives

$$
\begin{aligned}
-\frac{\partial V_{i} / \partial y}{\partial V_{i} / \partial w_{i}} & \geq \frac{p_{i} \mathbf{E}\left[\frac{\partial u_{i}\left(w_{i}-\tilde{\ell}_{i}, \hat{e}_{i}\right)}{\partial w_{i}^{f}} \mid y\right] \frac{\partial}{\partial y} \mathbf{E}\left(\tilde{\ell}_{i} \mid y\right)}{\left(1-p_{i}\right) \frac{\partial u_{i}\left(w_{i}, \hat{e}_{i}\right)}{\partial w_{i}^{f}}+p_{i} \mathbf{E}\left[\frac{\partial u_{i}\left(w_{i}-\tilde{\ell}_{i}, \hat{e}_{i}\right)}{\partial w_{i}^{f}} \mid y\right]} \\
& >p_{i} \frac{\partial}{\partial y} \mathbf{E}\left(\tilde{\ell}_{i} \mid y\right)
\end{aligned}
$$


because

$$
\mathbf{E}\left[\frac{\partial u_{i}\left(w_{i}-\tilde{\ell}_{i}, \hat{e}_{i}\right)}{\partial w_{i}^{f}} \mid y\right]>\frac{\partial u_{i}\left(w_{i}, \hat{e}_{i}\right)}{\partial w_{i}^{f}}
$$

Q.E.D.

\section{References}

Arnott, R. J., 1992, Moral Hazard and Competitive Insurance Markets, in: G. Dionne, ed., Contributions to Insurance Economics (Norwell, MA: Kluwer Academic Publishers).

Blomquist, G. C., 1988, The Regulation of Motor Vehicle and Traffic Safety (Norwell, MA: Kluwer Academic Publishers).

Boardman, A. E., D. H. Greenberg, A. R. Vining, and D. L. Weimer, 2001, Cost-Benefit Analysis, Concepts and Practice, 2nd edition (Englewood Cliffs, NJ: Prentice-Hall).

Caballé, J., and A. Pomansky, 1996, Mixed Risk Aversion, Journal of Economic Theory, 71: 485-513.

Chirunko, R. S., and E. P. Harper, Jr., 1993, Buckle Up or Slow Down? New Estimates of Offsetting Behavior and Their Implications for Automobile Safety Regulation, Journal of Policy Analysis and Management, 12, 270-296.

Dachraoui, K., G. Dionne, L. Eeckhoudt, and P. Godfroid, 2000, Proper Risk Behavior, Mimeo, HEC-Montréal.

Dionne, G., 1982, Moral Hazard and State Dependent Utility Function, Journal of Risk and Insurance, 49: 405-423.

Gollier, C., 2001, The Economics of Risk and Time (Cambridge, MA: MIT Press).

Graham, J. D., and S. Garber, 1984, Evaluating the Effects of Automobile Safety Regulation, Journal of Policy Analysis and Management, 3(2): 206-224.

Janssen, W. H., and E. Tenkink, 1988, Considerations on Speed Selection and Risk Homeostasis in Driving, Accident Analysis and Prevention, 20(2): 137-142.

Jansson, J. O., 1994, Accident Externality Charges, Journal of Transport Economics and Policy, Jan: 31-43.

Keeler, T. E., 1994, Highway Safety, Economic Behavior and Driving Environment, American Economic Review, 84: 684-693.

Miller, T. R., 1993, Costs and Functional Consequences of US Roadway Crashes, Accident Analysis and Prevention, 25(5): 593-607.

OECD, 1990, Behavioral Adaptations to Changes in the Road Transport System (Chicago: University of Chicago Press).

Peltzman, S., 1975, The Effects of Automobile Safety Regulation, Journal of Political Economy, 83(4): 677-725.

Peterson, S., G. Hoffer, and E. Millner, 1995, Are Drivers of Air-Bag-Equipped Cars More Aggressive? A Test of the Offsetting Behavior Hypothesis, Journal of Law and Economics, XXXVIII: 251-265.

Rothschild, M., and J. Stiglitz, 1970, Increasing Risk: I. A Definition, Journal of Economic Theory, 2: 225-243. 
Small, K., and J. A. Gomez-Ibanez, 1999, Urban Transportation, in: P. Cheshire and E. S. Mills, eds., Handbook of Regional and Urban Economics, vol 3: Applied Urban Economics (Amsterdam: North-Holland), pp. 1937-1999.

Viscusi, W. K., 1993, The Value of Risks to Life and Health, Journal of Economic Literature, 31(4): 1912-1946.

Wilde, G. J. S., 1982, The Theory of Risk Homeostasis: Implications for Safety and Health, Risk Analysis, 4: 209-225.

Wilde, G. J. S., 2001, Target Risk 2: A New Psychology of Safety and Health (Toronto, ON: PDE Publications). 